\title{
Trends in
}

\section{Ecology \& Evolution}

\section{Letter}

Inherited Gene

Regulation Unifies

Molecular

Approaches to

Nongenetic

Inheritance:

Response to

Edelaar et al.

Irene Adrian-Kalchhauser (1D, , , Sonia E. Sultan, ${ }^{2}$ Lisa Shama, ${ }^{3}$ Helen Spence-Jones, ${ }^{4}$

Stefano Tiso, ${ }^{5}$

Claudia Isabelle Keller Valsecchi, ${ }^{6}$ and Franz J. Weissing ${ }^{5}$

Our recent Opinion in TREE [1] on nongenetic inheritance presented insights gained through crosstalk between molecular and evolutionary biologists. Reviewing the molecular aspects of nongenetic inheritance, we identified broadly shared features and concluded that these 'epigenetic' mechanisms are more complex, functionally diverse, and variable across organisms than is commonly recognized. Despite a shared interest in transgenerational effects on gene expression and, hence, progeny phenotypes, the usage of current terminology (e.g., 'epigenetics') differs considerably among scientific disciplines. To highlight this shared focus, we proposed the unifying concept of 'inherited gene regulation' (IGR), which, with few exceptions (e.g., prions), provides an adequate functional description of the spectrum of molecular nongenetic inheritance systems. As specified in the article [1], IGR encompasses diverse genomeassociated mechanisms, such as DNA methylation and histone modifications, as well as cytoplasmic signaling components, including noncoding RNAs and hormones. By providing a simplified yet mechanistically accurate way to understand the molecular aspects of nongenetic inheritance, our paper aimed to help integrate these fascinating systems into evolutionary biology and ecology.

Our intention was not to subsume all forms of nongenetic inheritance under the IGR concept. For example, cultural inheritance may involve gene regulation during memory formation in the brain, but it would be a case of extreme (and misleading) reductionism to characterize cultural transmission as inherited gene regulation. Similarly, ecological inheritance cannot be reduced to inherited gene regulation even though it may create abiotic or biotic conditions that influence progeny gene expression. We intended to make clear our specific aims by stating that the scope of our article was limited to molecular systems, and by explicitly excluding cultural and ecological inheritance. In retrospect, we could have emphasized even more strongly that the focus of our article was specifically on molecular mechanisms and not on other systems of nongenetic inheritance, which we believe would require a substantially longer paper addressing some very different issues. We fully agree with the response of
Edelaar and colleagues [2]. We thank them for initiating this constructive dialog and for providing the opportunity to further clarify the scope of IGR. Our paper was intended as one step toward a larger goal that we share with our responding colleagues, and with the entire community of researchers who are expanding studies of inheritance processes beyond 'genes only': building a richer understanding of nongenetic inheritance in all its dimensions. Moving forward, we encourage further 'crosstalk' among diverse areas of expertise and perspectives in pursuit of this goal.

${ }^{1}$ Centre for Fish and Wildlife Health, Department for Infectious Diseases and Pathobiology, Vetsuisse Faculty, University of Bern, Bern 3012, Switzerland

${ }^{2}$ Biology Department, Wesleyan University, Middletown, CT 06459, USA

${ }^{3}$ Coastal Ecology Section, Alfred Wegener Institute Helmholtz Centre for Polar and Marine Research, List 25992, Germany ${ }^{4}$ Centre for Biological Diversity, School of Biology, University of St Andrews, St Andrews KY16 9TF, UK

${ }^{5}$ Groningen Institute for Evolutionary Life Sciences, University of Groningen, Groningen, 9747, The Netherlands

${ }^{6}$ Institute of Molecular Biology (IMB), Mainz 55128, Germany

${ }^{*}$ Correspondence:

irene.adrian-kalchhauser@vetsuisse.unibe.ch

(I. Adrian-Kalchhauser).

https://doi.org/10.1016/j.tree.2021.03.004

(c) 2021 Elsevier Ltd. All rights reserved.

\section{References}

1. Adrian-Kalchhauser, I. et al. (2020) Understanding 'Nongenetic' inheritance: insights from molecular-evolutionary crosstalk. Trends Ecol. Evol. 35, 1078-1089

2. Edelaar, P. et al. (2021) Inherited-gene-regulation is not enough to understand non-genetic inheritance. Trends Ecol Evol. Published online March 23, 2021. https://doi.org/ 10.1016/.tree.2021.03.002 